\title{
The effectiveness of cognitive behavioral therapy with respect to psychological symptoms and recovering autobiographical memory in patients suffering from post-traumatic stress disorder
}

\author{
This article was published in the following Dove Press journal: \\ Neuropsychiatric Disease and Treatment \\ 19 February 2015 \\ Number of times this article has been viewed
}

\author{
Fatemehsadat Akbarian' \\ Hafez Bajoghli ${ }^{2,3}$ \\ Mohammad Haghighi ${ }^{4}$ \\ Nadeem Kalak ${ }^{5}$ \\ Edith Holsboer-Trachsler ${ }^{5}$ \\ Serge Brand $d^{5,6}$ \\ 'Psychology and Counseling \\ Organization of Iran, Science and \\ Research Branch, Islamic Azad \\ University, Tehran, Iran; ${ }^{2}$ Iranian \\ National Center for Addiction \\ Studies (INCAS), Iranian Institute \\ for Reduction of High-Risk Behaviors, \\ Tehran University of Medical Sciences, \\ Tehran, Iran; ${ }^{3}$ ASEAN Institute \\ for Health Development, Mahidol \\ University, Nakhonpathom, Thailand; \\ ${ }^{4}$ Research Center for Behavioral \\ Disorders and Substances Abuse, \\ Hamadan University of Medical \\ Sciences, Hamadan, Iran; ${ }^{5}$ Center for \\ Affective, Stress and Sleep Disorders, \\ Psychiatric Clinics of the University of \\ Basel, Basel, Switzerland; ' $D$ epartment \\ of Sport, Exercise and Health, Sport \\ Science Section, University of Basel, \\ Basel, Switzerland
}

Objectives: Given the persistence of post-traumatic stress disorder (PTSD) and its major impact on everyday life, it is important to identify effective treatments. In additional to pharmacological treatments, psychotherapeutic treatments are also highly effective. The aim of the present study was to investigate, among a sample of patients suffering from PTSD, the influence of an additional cognitive behavioral therapy (CBT) intervention on their symptoms of PTSD, depression, and anxiety, and on autobiographical memory.

Methods: A total of 40 patients suffering from PTSD (mean age: 31.64 years; $78.6 \%$ female patients) and under psychopharmacological treatment were randomly assigned to an intervention or control condition. The intervention consisted of ten group sessions (one 60-90 minute session per week) of CBT. At baseline and 10 weeks later, a series of self-rating and experts'rating questionnaires were completed.

Results: Over time, symptoms of PTSD, depression, and anxiety decreased; however, greater improvement was observed in the experimental than the control condition. Likewise, as a general pattern of results, memory performance improved over time, though again this improvement was greater in the experimental condition.

Conclusion: Compared to a control condition, additional CBT improves the treatment of PTSD, with respect to both symptoms and autobiographical memory.

Keywords: anxiety, depression, PTSD, autobiographical memory

\section{Objectives}

Post-traumatic stress disorder (PTSD) is a common psychiatric disorder that may occur following a range of experiences including witnessing life-threatening incidents such as in a war zone, natural disasters, terror attacks, and serious car accidents. Persistent avoidance of stimuli, slow responses (eg, feelings of isolation and loss of personal interests), re-experiencing the trauma (unavoidable recall of the traumatic event, nightmares), and increased autonomic stimulation (hyperactivity, irritability, and sleep disorders) are the main symptoms of this condition, observable shortly after onset. ${ }^{1}$ Whereas not everyone exposed to such life events develops PTSD, in the USA, it occurs among $10 \%$ of women and $5 \%$ of men who were previously exposed to such kind of live events. ${ }^{2}$ Moreover, the life-time risk in the general population of developing at least one episode of PTSD is estimated to be $8 \%-9 \%{ }^{3}$ In addition to flashbacks, nightmares are often reported by those suffering from PTSD. In this context, sleep disorders comprise about $70 \%$ of the complaints of patients with PTSD. ${ }^{4,5}$ They are also more likely to commit suicide than persons without PTSD. ${ }^{6,7}$
Correspondence: Serge Brand Center for Affective, Stress and Sleep Disorders, Psychiatric Clinics of the University of Basel, Wilhelm Klein-Strasse 27, 4012 Basel, Switzerland

$\mathrm{Tel}+4|6| 3255 \mid$ I4

Fax $+4|6| 3255513$

Email serge.brand@upkbs.ch 
The risk of suicide among those with PTSD is higher for people reporting nightmares than for those who do not. ${ }^{8}$ Accordingly, symptoms need to be recognized and treated appropriately if they are not to become persistent. ${ }^{9}$ In addition, patients with PTSD are at high risk of developing comorbid psychiatric disorders such as mood, anxiety, and substance abuse disorders. Furthermore, the high chronicity, severity, and comorbidity of PTSD are associated with high levels of functional and psychosocial disability, ${ }^{10}$ and also with dramatic impairments in memory and autobiographical memory. ${ }^{11}$ Accordingly, in the present study, we investigated the influence of additional CBT on the autobiographical memory of patients with PTSD.

As regards treatment interventions, PTSD can be treated both pharmacologically and non-pharmacologically; that is, psychotherapeutically. Depending on symptom severity and comorbid disorders, pharmacological treatment typically involves administration of selective serotonin reuptake inhibitors (SSRIs), benzodiazepines, and antipsychotics. Diverse medication classes, including antidepressants, anticonvulsants, antipsychotics and antiadrenergic drugs have been used to treat PTSD. Thereby, antidepressants are the most used and prescribed medication for PTSD, ${ }^{3}$ and SSRIs are the only class of medication approved for treatment of PTSD in the USA. SSRIs seem to be safer and better tolerated than other psychotropic medications and many of the clinical trials showed that there is evidence of best efficacy in reducing PTSD symptoms for this class of medication. ${ }^{3}$ SSRIs have shown to be efficient in reducing symptom severity and in preventing PTSD patients from relapsing, although just a few patients (about 60\%) respond to treatment and only approximately $20 \%-30 \%$ have been fully remitted. ${ }^{12}$ SSRI paroxetine, for example, succeeded in effective reduction of symptoms of all three PTSD symptom clusters, namely re-experiencing of the trauma, increased arousal, and avoidance of situations that resemble the traumatic event. ${ }^{10}$ Among the empirically-supported psychotherapeutic interventions are trauma-focused cognitive behavioral therapy (CBT), exposure-based therapy, eye movement desensitization and reprocessing, ${ }^{10}$ stress inoculated training, and CBT. ${ }^{12}$ Further, prolonged exposure is the most researched and best validated non-pharmacological treatment for PTSD. ${ }^{3}$ In the present study, we focused on CBT.

Several placebo-controlled trials and meta-analyses support the view that these non-pharmacological treatments are effective in reducing PTSD symptoms. ${ }^{13}$ International guidelines recommend non-pharmacological treatment for all PTSD patients as the first option (Polak et $\mathrm{al}^{10}$ ) and pharmacological treatment as a second option, but no consensus has been achieved on these alternatives so far. ${ }^{3}$ More specifically, and contrary to Polak et al, ${ }^{10}$ Ipser et al ${ }^{14}$ concluded based on a Cochrane review that medication treatments can be effective in treating PTSD, and should be considered as a part of the treatment of PTSD, with the application SSRIs as first-line agents in the pharmacotherapy of PTSD. However, we also note that the Institute of Medicine Report concluded that there was no evidence that one of the several medication classes was effective for treatment of PTSD, while the UK National Institute for Clinical Excellence recommended in 2005 that medications should only be used as a second-line treatment for PTSD. ${ }^{3}$ This may also be due to the much higher treatment costs for pharmacotherapies in comparison to psychotherapies, especially in relation to higher expected relapse rates with pharmacotherapies. ${ }^{10}$ Moreover, the efficacy of pharmacological treatment for PTSD may also depend on the type of symptoms. On the other hand, and apparently paradoxically, Mellman et al ${ }^{16}$ estimated that $77 \%$ of PTSD patients in a community-based sample received pharmacotherapy. This high rate for pharmacotherapy may reflect the low availability and accessibility of psychotherapy. ${ }^{10}$ There is also some evidence that those patients who report more severe sleep difficulties have poorer treatment outcomes with psychotherapy. ${ }^{3}$

Last, combined pharmacotherapy and psychological therapies are especially recommended when patients have not responded to either intervention alone, ${ }^{16}$ and this needs to be further examined in future research. ${ }^{18,19}$

A further issue of PTSD regards memory performance, in that one of the most serious and frequent symptoms of PTSD is intrusive memories of the traumatic event. ${ }^{20}$ Since PTSD symptoms often persist long after exposure to the traumatic event, researchers have given particular attention to memory as a key factor in the pathogenesis of the disorder. ${ }^{22}$ Most strikingly, there is evidence that PTSD patients show difficulties in retrieval of autobiographical memories. ${ }^{21}$ In addition, patients with PTSD tend to report more memories related to the traumatic event compared to people without PTSD. ${ }^{20}$ They tend to exhibit difficulties in recalling specific memories; producing a so-called "overgeneralized" autobiographical memory.

Furthermore, it is not only autobiographical memories that are characterized by overgeneralization. Expectations that individuals with PTSD have about imagined future events are also characterized by overgeneralization. ${ }^{20}$ Overgeneralized autobiographical memory has been implicated as an important mechanism underlying the 
pathogenesis of PTSD, and the close connection between overgeneralization in autobiographical memory and in thinking about the future seems to be conducive to the onset and maintenance of the disorder. ${ }^{22}$ To summarize, PTSD is a severe psychiatric disorder, affecting patients' emotions, behavior, social life, and cognitions. With respect to cognitions, memory performance, and more specifically, autobiographical memory performance, is impaired. PTSD is treated pharmacologically and non-pharmacologically. From previous research, we learn that the effect of additional CBT in combination with psychopharmacological treatment is poorly investigated, and in particular that the influence of additional CBT on autobiographical memory has not so far been investigated. Accordingly, the aims of the present study were to investigate the influence of additional CBT on symptoms of PTSD, depression, and anxiety, and on autobiographical memory in patients suffering from PTSD, as compared to a control condition. We hold that the present study has the potential to expand on previous research in that the data from both the case-control design and the study targets should add to the current literature in an important way.

The following two hypotheses were formulated. First, following Hendriksen et $a{ }^{12}$ Polak et $\mathrm{al}^{10}$ and others, we expected improved symptoms of PTSD, depression, and anxiety in the CBT as compared to the control group. Second, following Brown et $\mathrm{al}^{21}$ we expected that autobiographic memory performance would be low at baseline and would improve in the treatment as compared to the control group. Additionally, given the lack of previous research, we treated exploratory potential changes in autobiographical memory as a function of domain (semantic and episodic memory) and developmental period (childhood, adulthood, and recent life).

\section{Methods \\ Procedure}

Patients clinically diagnosed as suffering from PTSD (accident-related injuries, cancer, domestic violence) participated in this study. They were all recruited from the Sina Hospital, Mahak (cancer foundation), and from psychiatric and psychological centers in Tehran, Iran. Before entering the study, patients were fully informed about its aims, scope, and procedures and gave written informed consent. Additionally, the local ethics committee approved the study, and the entire study was performed according to the rules laid down in the Declaration of Helsinki.

After a thorough psychiatric interview, all patients were psychopharmacologically treated with SSRIs, neuroleptics, or benzodiazepines (Table 1). Dosages were individually adapted to patients and kept constant throughout the study in order to achieve and maintain treatment efficacy. Next, patients were randomly assigned either to an intervention or control condition. At the beginning and at the end of the study 10 weeks later, patients completed a series of questionnaires as described in more details below, and experts' ratings were performed in parallel. In addition to the diagnostic interview, both groups had the autobiographical memory interview. After completion of the study, patients in the control condition received the same treatment as that provided for the intervention group. Psychologists and psychiatrists involved in the study and responsible for experts' ratings were not aware of patients' group assignment.

Table I Descriptive and statistical overview of patients' sociodemographic dimensions and medication at baseline, separately by group (intervention versus control condition)

\begin{tabular}{|c|c|c|c|}
\hline \multirow[t]{3}{*}{$\mathbf{N}$} & \multicolumn{2}{|l|}{ Group } & \multirow[t]{3}{*}{ Statistics } \\
\hline & Intervention group & Control group & \\
\hline & 20 & 20 & \\
\hline Age, years (standard deviation) & $32.07(5.76)$ & $31.21(6.10)$ & $t(38)=0.38, P>0.78$ \\
\hline Sex (male/female) & $5 / 15$ & $7 / 13$ & $\chi^{2}(d f=I, N=40)=0.85, P>0.35$ \\
\hline Civil status (single/married) & $6 / 14$ & $7 / 13$ & $\chi^{2}(I)=0.19, P>0.65$ \\
\hline \multicolumn{4}{|l|}{ Medications } \\
\hline Benzodiazepines (yes/no) & $12 / 8$ & $10 / 10$ & $\chi^{2}(I)<I .5, P>0 . I$ \\
\hline Antidepressants (yes/no) & $18 / 2$ & $16 / 4$ & $\chi^{2}(I)<I .5, P>0.1$ \\
\hline Neuroleptics (yes/no) & $6 / 14$ & $9 / 11$ & $\chi^{2}(I)<I .5, P>0.1$ \\
\hline $\begin{array}{l}\text { Patients receiving one } \\
\text { pharmacologic agent (yes/no) }\end{array}$ & $20 / 0$ & $20 / 0$ & - \\
\hline $\begin{array}{l}\text { Patients receiving two } \\
\text { pharmacologic agents }\end{array}$ & $9 / 11$ & $11 / 9$ & $\chi^{2}(I)<1.0, P>0.1$ \\
\hline $\begin{array}{l}\text { Patients receiving three or more } \\
\text { pharmacologic agents }\end{array}$ & $3 / 17$ & $2 / 18$ & $\chi^{2}(I)<I .0, P>0.1$ \\
\hline
\end{tabular}




\section{Randomization}

First, a ballot box containing two different colored chips was prepared (20 blue chips for the intervention, and 20 red chips for the control condition). A psychologist (FA) drew chips consecutively and assigned patients to either to the intervention or control condition. FA was also responsible for the psychotherapeutic intervention, though she was not further involved in experts' ratings.

\section{Sample}

Patients suffering from PTSD and attending the Sina Hospital, Mahak (cancer foundation), and other psychiatric and psychological centers in Tehran, Iran were approached regarding the study. The CONSORT flow chart (Figure 1) indicates how many patients were approached, and how many completed the study. Of the 76 patients approached, $40(52.63 \%)$ agreed to participate in the study. Further, 12 patients, who were randomly assigned to the intervention or the control condition, withdraw after randomization and baseline assessment; accordingly, 28 patients started the study. Of these, six $(21.43 \%)$ were male (mean age: 29.50 years, standard deviation: 4.97$)$ and 22 (78.57\%) were female (mean age: 32.23 years, standard deviation: 6.02; $t[26]=1.02, P=0.32, d=0.496)$. Please note that to perform the statistical analysis, the intent-to-treat algorithm with the last observation carried forward procedure was performed, and not the per protocol algorithm (see also the "Statistical analysis" section at the end of the "Methods" section).

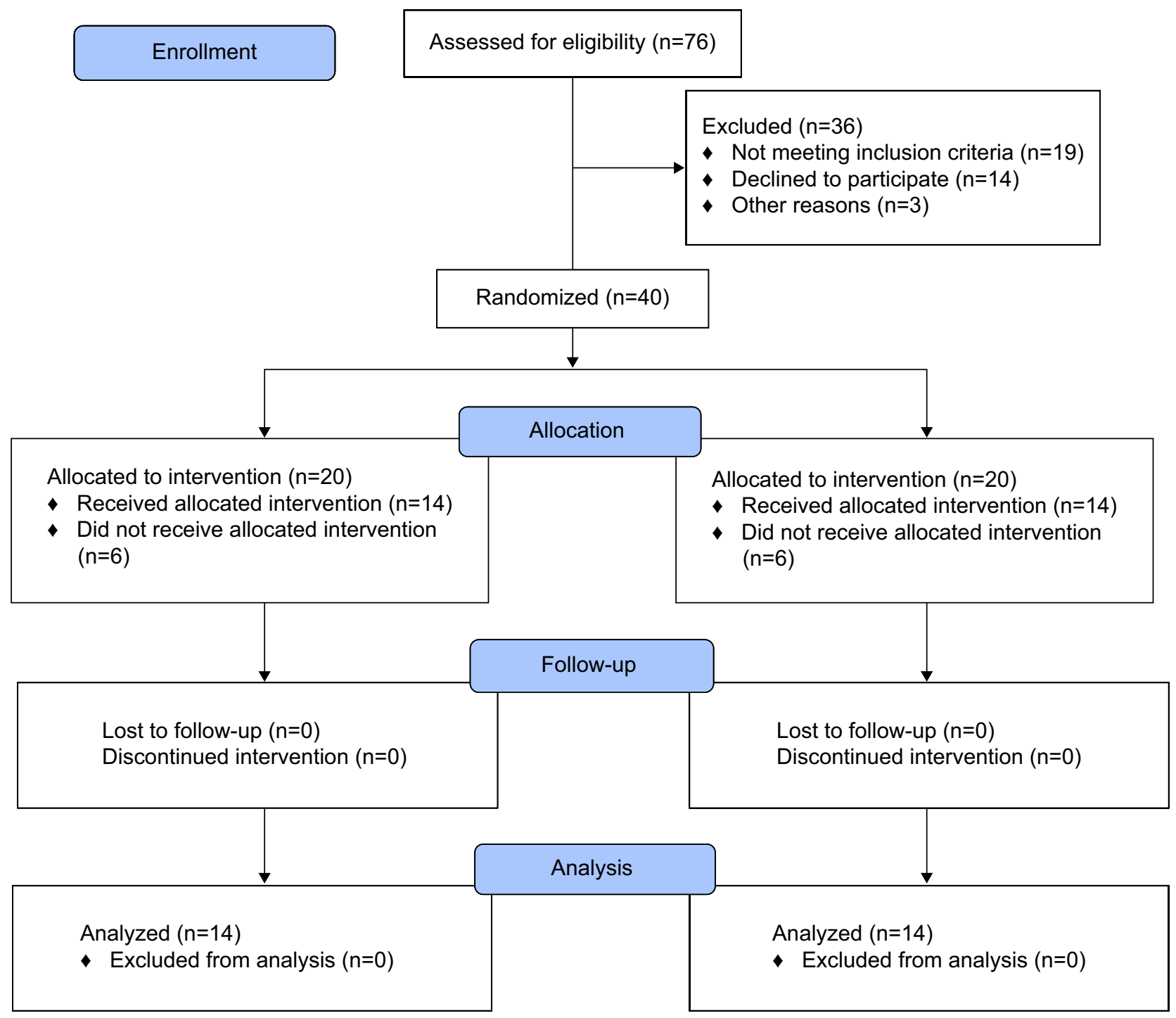

Figure I The CONSORT flow diagram shows the number of patients enrolled, randomly assigned to the study conditions, and finally analyzed. 
Inclusion criteria were as follows: 1) a diagnosis of PTSD according to the diagnostic criteria of the $D S M-5,23$ and 2) aged between 18 and 45 years. Exclusion criteria were the following: 1) further psychiatric comorbidities such as major depressive disorder, anxiety disorders, substance abuse (alcohol, drugs), psychosis, and personality disorders; 2) women who were pregnant or intending to get pregnant or who were breast-feeding; 3) known physical illness such as heart disease; and 4) patients already undergoing a psychotherapeutic treatment. Patients were also excluded if they decided to withdraw from the study (though without indicating the reasons).

Sample characteristics are reported separately for intervention and control groups in Table 1.

No differences between the intervention and the control group were observed as regards sociodemographic variables and medications (types and number of medications; for symptoms at baseline for PTSD, depression, and anxiety, Table 2).

\section{Materials}

One questionnaire was employed, which covered demographic variables such as sex, age, civil status, educational level, and current employment.

\section{Symptoms of PTSD}

To assess symptoms of PTSD, the Impact of Event ScaleRevised ${ }^{24}$ questionnaire was employed. It consists of 22 items asking about PTSD symptoms such as avoidance, arousal, and unpleasant thought. Answers are given on 5-point Likertscales ranging from 0 (never) to 4 (extremely), with higher sum scores reflecting more severe symptoms (Cronbach's alpha $=0.92$ ).

\section{Symptoms of depression}

To assess symptoms of depression, the Beck Depression Inventory ${ }^{25}$ questionnaire was employed. It consists of 21 items asking about symptoms of depression such as pessimism and negative thoughts, sadness, reduced incentives, and physiological problems. Answers are given on 4-point Likert-scales ranging from 0 (never) to 3 (extremely), with higher sum scores reflecting more severe symptoms (Cronbach's alpha $=0.87$ ).

\section{Symptoms of anxiety}

To assess symptoms of anxiety, the Beck Anxiety Inventory ${ }^{26}$ questionnaire was employed. It consists of 21 items asking about symptoms of anxiety such as worry, anxiety, and physiological problems. Answers are given on 4-point Likertscales ranging from 0 (never) to 3 (extremely), with higher sum scores reflecting more severe symptoms (Cronbach's alpha $=0.92$ ).

\section{Assessing semantic and episodic memory performance}

To assess autobiographical memory, both at baseline and at follow-up, an autobiographical memory interview ${ }^{27}$ was performed. The face-to-face interview consists of 52 items and assesses semantic and episodic memory performance separately for childhood, adulthood, and current life. Typical items are: "Mention three of your childhood friends' or neighbors' full names", "Mention a memory form that course or that job", and "Name a place you visited last

Table 2 Descriptive and statistical overview of patients' symptom-related dimensions, separated by group (intervention versus control condition) and time (baseline versus follow-up)

\begin{tabular}{|c|c|c|c|c|c|c|c|}
\hline & \multicolumn{7}{|l|}{ Time points } \\
\hline & \multirow{2}{*}{\multicolumn{2}{|c|}{$\begin{array}{l}\text { Baseline } \\
\text { Group }\end{array}$}} & \multirow{2}{*}{\multicolumn{2}{|c|}{$\begin{array}{l}\text { Follow-up } \\
\text { Group }\end{array}$}} & \multirow{3}{*}{$\begin{array}{l}\text { Time } \\
\boldsymbol{F ( \eta ^ { 2 } )}\end{array}$} & \multicolumn{2}{|l|}{ Statistics } \\
\hline & & & & & & Group & \multirow{2}{*}{$\begin{array}{l}\text { Time } \times \text { group } \\
\text { intervention } \\
F\left(\eta^{2}\right)\end{array}$} \\
\hline & $\begin{array}{l}\text { Intervention } \\
\text { group }\end{array}$ & $\begin{array}{l}\text { Control } \\
\text { group }\end{array}$ & $\begin{array}{l}\text { Intervention } \\
\text { group }\end{array}$ & $\begin{array}{l}\text { Control } \\
\text { group }\end{array}$ & & $F\left(\eta^{2}\right)$ & \\
\hline Symptoms of depression & $66.43(5.26)$ & $64.64(5.58)$ & $38.93(2.92)$ & $43.64(4.29)$ & $336.69 * * *(0.93)[\mathrm{L}]$ & $\mathrm{I} .63(0.059)[\mathrm{S}]$ & $6.05 *(0.189)[\mathrm{L}]$ \\
\hline Symptoms of anxiety & $46.29(6.39)$ & $46.86(6.8 I)$ & $42.14(4.85)$ & $46.21(5.79)$ & $20.59 * * *(0.446)[\mathrm{L}]$ & $\mathrm{I} .0 \mathrm{I}(0.04 \mathrm{I})[\mathrm{S}]$ & $1 \mathrm{I} .2 \mathrm{I}^{* *}(0.30 \mathrm{I})[\mathrm{L}]$ \\
\hline \multicolumn{8}{|l|}{ Symptoms of PTSD } \\
\hline Total score & $66.36(4.8 I)$ & $64.00(5.11)$ & $55.57(4.77)$ & $63.50(5.68)$ & $27.80 * * *(0.5 \mid 7)[\mathrm{L}]$ & $3.00(0.102)[\mathrm{M}]$ & $23.09 * * *(0.470)[\mathrm{L}]$ \\
\hline Avoidance & $22.50(3.92)$ & $22.14(4.09)$ & $18.79(2.75)$ & $21.43(2.90)$ & $8.31 *(0.242)[L]$ & $1.16(0.043)[\mathrm{S}]$ & $3.81(0.128)[\mathrm{M}]$ \\
\hline Intrusion & $21.71(3.69)$ & $20.43(3.34)$ & I8.43 (2.7I) & $20.64(2.56)$ & $7.2 I^{*}(0.25 \mathrm{I})[\mathrm{L}]$ & $0.025(0.00 \mathrm{I})[\mathrm{S}]$ & $9.24 * *(0.262)[\mathrm{L}]$ \\
\hline Hyperarousal & $22.21(1.63)$ & $22.07(1.14)$ & $18.38(2.65)$ & $21.43(2.17)$ & $22.08 * * *(0.459)[\mathrm{L}]$ & $6.48 *(0.199)[\mathrm{L}]$ & II.26** (0.302) [L] \\
\hline
\end{tabular}

Notes: Degrees of freedom: always $(\mathrm{I}, 38) ; * P<0.05 ; * * P<0.01 ; * * * P<0.00$ I. Effect sizes $\left(\eta^{2}\right)$ : $[\mathrm{S}]=$ small effect; $[\mathrm{M}]=$ medium effect; $[\mathrm{L}]=$ large effect.

Abbreviation: PTSD, post-traumatic stress disorder. 
year." Trained psychologists and psychiatrists not otherwise involved in the treatment and scoring assessed the semantic and episodic memory performance.

\section{Intervention}

The intervention was conceptualized on the basis of Clark and Beck's CBT for the treatment of PTSD ${ }^{28}$ and involved the following procedures. Trained and experienced psychotherapists (psychologists, psychiatrists) not involved in the experts' ratings performed the intervention. Group sessions took place once per week for 60-90 minutes.

\section{First session}

The first session involves establishing a therapeutic relationship with the client: the therapist introduces and explains PTSD, together with its signs and symptoms; brochures are provided about the disease commensurate with the knowledge and educational level of clients. CBT is explained in relation to cognitive features of PTSD, including thoughts, negative beliefs and memories related to the event, negative emotions, and avoidance of traumarelated memories.

\section{Second session}

The second session involves assessment of thoughts and negative beliefs about PTSD and cognitive assessment and rehabilitation of PTSD including correction of erroneous assessments. It also includes modification and cognitive rehabilitation of incorrect assessments.

\section{Third session}

The third session involves cognitive restructuring with a focus on trauma and identification of beliefs and negative evaluations of clients, in cases of injury, and its consequences for explaining the emergence of PTSD.

\section{Fourth session}

The fourth session involves identifying and correcting a patient's faulty thinking and assessment; modifying it through the use of techniques such as reviewing the evidence, analysis of profit and loss, and identifying and modifying cognitive errors; and setting homework.

\section{Fifth session}

The fifth session involves evaluating assignments related to the previous session, including the technique of reviewing the evidence, and explaining new techniques such as loss and profit techniques.

\section{Sixth and seventh session}

The sixth and seventh session involve imagined flooding.

\section{Eighth and ninth session}

The eighth and ninth session involve maladaptive cognitive and behavioral modification techniques through mindfulness training.

\section{Tenth and closing session}

The tenth and closing session include control of emotion through progressive muscle relaxation techniques with breathing regulation.

Individualized health plans were provided in sessions of 1 hour per week in medical centers.

\section{Control condition}

Once a week, patients in the control condition individually attended the center for a medical check. Further, medication regimen was asked about as well as possible side effects. Patients, however, were free to talk about their current life, concerns, and disorders. On doing so, we kept social contact with the professional hospital staff equal between the intervention and control condition.

\section{Statistical analysis}

All computations were performed per intention-to-treat with the last-observation-carried-forward $(\mathrm{N}=20$ per group). A series of analyses of variance for repeated measures was performed with the factors "time" (baseline, follow-up) and "group" (intervention versus control group), and the dependent variables of symptoms of PTSD, depression, anxiety, and memory performance. For analyses of variance, effect sizes are indicated with the partial eta squared $\left(\eta^{2}\right)$, with $0.059 \geq \eta^{2} \geq 0.01$ indicating small, $0.139 \geq \eta^{2} \geq 0.06$ indicating medium, and $\eta^{2} \geq 0.14$ indicating large effect sizes. The level of significance was set at $P \leq 0.05$, and all statistics were processed using SPSS $^{\circledR} 20.0$ (IBM Corporation, Armonk, NY, USA) for Apple McIntosh ${ }^{\circledR}$.

\section{Results}

All descriptive and statistical information (means, standard deviations, $P$-values, effect sizes) are reported in the tables and therefore not repeated in the text.

Table 2 gives a descriptive and statistical overview of symptom-related dimensions separately by group (intervention versus control condition) and time (baseline versus follow-up). Symptoms of both depression and anxiety 
Table 3 Descriptive and statistical overview of patients' memory performance, separated by group (intervention versus control condition) and time (baseline versus follow-up)

\begin{tabular}{|c|c|c|c|c|c|c|c|}
\hline & \multicolumn{7}{|l|}{ Time points } \\
\hline & \multirow{2}{*}{\multicolumn{2}{|c|}{$\frac{\text { Baseline }}{\text { Group }}$}} & \multirow{2}{*}{\multicolumn{2}{|c|}{$\frac{\text { Follow-up }}{\text { Group }}$}} & \multirow{3}{*}{$\begin{array}{l}\text { Time } \\
F\left(\eta^{2}\right)\end{array}$} & \multicolumn{2}{|l|}{ Statistics } \\
\hline & & & & & & \multirow{2}{*}{$\begin{array}{l}\text { Group } \\
F\left(\eta^{2}\right)\end{array}$} & \multirow{2}{*}{$\begin{array}{l}\text { Time } \times \text { group } \\
\text { interaction } \\
F\left(\eta^{2}\right)\end{array}$} \\
\hline & $\begin{array}{l}\text { Intervention } \\
\text { group } \\
\text { M }\end{array}$ & $\begin{array}{l}\text { Control } \\
\text { group } \\
\text { (SD) }\end{array}$ & $\begin{array}{l}\text { Intervention } \\
\text { group } \\
\text { M }\end{array}$ & $\begin{array}{l}\text { Control } \\
\text { group } \\
\text { (SD) }\end{array}$ & & & \\
\hline \multicolumn{8}{|l|}{ Childhood memory } \\
\hline Semantic memory & $16.18(2.22)$ & I5.64 (2.5I) & $16.21(1.49)$ & I5.79 (2.58) & $0.32(0.01)[S]$ & $0.35(0.0 \mathrm{I} \mid 3)[\mathrm{S}]$ & $0.12(0.004)[S]$ \\
\hline Episodic memory & $3.68(0.95)$ & $4.04(1.25)$ & $3.85(0.63)$ & $4.18(1.14)$ & $\mathrm{I} .88(0.067)[\mathrm{S}]$ & $0.02(0.00 \mathrm{I})[\mathrm{S}]$ & $0.85(0.032)[S]$ \\
\hline \multicolumn{8}{|l|}{ Adult memory } \\
\hline Semantic memory & $16.280(1.37)$ & $15.39(1.90)$ & I7.04 (I.22) & $15.05(1.37)$ & $10.73 * * *(0.292)[\mathrm{L}]$ & $6.03 * * *(0.188)[\mathrm{L}]$ & $4.36^{* * *}(0.144)[\mathrm{L}]$ \\
\hline Episodic memory & $4.10(1.23)$ & $3.68(1.29)$ & $4.7 I(0.85)$ & $3.89(1.15)$ & I4.54*** (0.359) [L] & $3.33^{*}(0.113)[\mathrm{M}]$ & $2.235(0.079)[\mathrm{M}]$ \\
\hline \multicolumn{8}{|l|}{ Recent memory } \\
\hline Semantic memory & I7.29 (2.68) & $16.9(3.07)$ & $17.86(2.21)$ & I6.75 (2.87) & $2.96 *(0.102)[\mathrm{M}]$ & $3.79 *(0.127)[\mathrm{M}]$ & $0.62(0.023)[S]$ \\
\hline Episodic memory & $4.00(1.30)$ & 3.29 (1.39) & $4.57(0.99)$ & $3.32(1.34)$ & $9.37^{* * *}(0.265)[\mathrm{L}]$ & $7.29 * * *(0.219)[\mathrm{L}]$ & $4.39 * *(0.144)[\mathrm{L}]$ \\
\hline
\end{tabular}

Notes: Degrees of freedom: always $(\mathrm{I}, 38) ; * P<0.05 ; * * P<0.01 ; * * * P<0.001$. Effect sizes $\left(\eta^{2}\right):[S]=$ small effect; $[M]=$ medium effect; $[\mathrm{L}]=$ large effect.

decreased over time. No group differences were observed. The significant time by group interactions showed that symptoms of depression and anxiety decreased significantly more in the intervention than in the control group.

For PTSDs, total score, avoidance, intrusion, and hyperarousal decreased over time. For hyperarousal, the control group had significantly higher scores than the intervention group. Medium to large effect sizes were observed for the time by group interaction; PTSD scores decreased more over time in the intervention than in the control group (Table 2).

\section{Autobiographical memory}

Table 3 gives a descriptive and statistical overview of symptom-related dimensions separately by group (intervention versus control condition) and time (baseline versus follow-up).

For childhood memory there were no significant changes over time or between groups.

For adult memory (semantic and episodic) scores improved over time but more in the experimental group.

For Recent memory, scores improved over time but improved more in the experimental group.

For positive scores (general), scores increased over time but declined more in the experimental group.

For positive scores (specific), scores increased over time with a greater increase in the experimental group.

\section{Discussion}

The key findings of the present study are that additional CBT significantly reduced symptoms of PTSD, depression, and anxiety among a sample of patients suffering from PTSD, as compared to those in a control condition. Moreover, results showed that CBT also improved autobiographical memory over time. The present study adds to the current literature in an important way in demonstrating the advantage of additional non-pharmacological treatments in patients suffering from PTSD; it significantly reduced symptoms and improved memory performance.

Two hypotheses were formulated, and these are now considered in turn. Our first hypothesis was that there would be greater symptom improvements in the intervention than the control group, and this hypothesis was fully supported. Accordingly, the present results are in accordance with a wealth of previous research that has shown a favorable influence of (additional) CBT in the treatment of PTSD, as compared to pharmacological treatment alone. ${ }^{10,12,17}$ However, the present study expands on previous research in showing that a highly standardized group therapy for ten sessions over a period of 10 weeks improved symptoms of PTSD, depression, and anxiety.

Our second hypothesis was that additional CBT would also improve dimensions of autobiographical memory, and this was broadly supported. Specifically, for autobiographical memory performance for adulthood and the current period of life, the group by time interaction showed that additional CBT led to a significant improvement for semantic memory (but not episodic memory) for adult memory, whereas for recent memory, additional CBT improved episodic memory (but not semantic memory). To the best of our knowledge, similar findings have not so far been reported; accordingly the present study expands upon existing research on PTSD in demonstrating that highly standardized CBT intervention 
has a positive influence not only on symptoms but also on autobiographical memory.

The present data do not allow any deeper understanding of the underlying cognitive-emotional and neurophysiological mechanisms. However, the following hypotheses are advanced.

As regards the cognitive-emotional changes, we note that the CBT program was specifically targeted at changing and favorably modifying dysfunctional thoughts (see "Methods" section). Accordingly, we assume that patients in the intervention group learned to restructure and positively alter their dysfunctional thoughts and emotions, which as a result reduced symptoms of depression, anxiety, and PTSD. To illustrate, by the second session, patients in the intervention group were already listing their thoughts and negative believes about PTSD and were evaluating the extent to which their behavior was causally associated with the dramatic event leading to PTSD. Likewise they were also evaluating the degree to which they were victims of the event, and the thoughts and feelings associated with it, and, in contrast, to what extent they could learn to manage, modify, and improve their thoughts. Connected to this, we believe that through the CBT intervention, patients became aware of their (unconscious) dysfunctional thoughts and beliefs and learned to evaluate the extent to which their thoughts and feelings were causally linked to the emergence and maintenance of symptoms of depression, anxiety, and PTSD, and that they learned how to cope with their current situation. On the flip side, we note that favorable changes in symptoms and memory were also observed in the control group. Therefore, the present data also show evidence that a mere psychopharmacological treatment leads to improvements both in symptoms and in memory (see also Polak et al, ${ }^{10}$ Ipser et $\mathrm{al}^{14}$ ).

To explain PTSD from a neuroendocrinologic point of view, it is assumed that an increase in the adrenergic activity of the central nervous system (CNS) is associated with the pathophysiology of PTSD and more specifically with sleep disorders. ${ }^{29}$ In fact, it seems that many of the symptoms of PTSD could be pathophysiologically caused or intensified by an increase in the adrenergic activity of the CNS. ${ }^{29,30}$ Such an increase in adrenergic activity can lead to increased secretion of norepinephrine and to elevated responsiveness of receptors to norepinephrine. Accordingly, though highly speculative, we suggest that CBT was able to decrease the adrenergic activity in the CNS. Likewise, though unfortunately not assessed, we believe that CBT was able to improve patients' sleep, which in turn may have led to improvement in symptoms. Indeed, there is a wealth of evidence for a close association between symptoms of depression and anxiety and poor sleep. ${ }^{31-33}$ Accordingly, it is highly conceivable that the sleep of patients in the intervention group improved, and in a sort of positive feedback loop this also ameliorated symptoms of depression, anxiety, and PTSD. Indeed, as regards the association between sleep and PTSD, specific alpha1receptor-blockers such as prazosin and specific H1-receptor blockers such as hydroxyzine reduce occurrence of nightmares and sleep disturbances among patients suffering from PTSD as compared to a control condition. ${ }^{35}$

Despite the intriguing results, several limitations warn against overgeneralizing the results. First, the sample size was rather small and there were drop-outs. However, the effect-size calculations which we report are not sensitive to sample sizes. Second, the age range was limited to below 45 years of age; we did this to keep the sample homogeneous and to avoid possible confounders such as age. Nevertheless, we acknowledge, that also enrolling elderly patients would have been advisable, especially given that an emphasis of the study was on autobiographical memory, which would be expected to be worse, and therefore possible more amenable to treatment, in older subjects. Third, sleep was not assessed though there is striking evidence that improved sleep is associated with improvements in symptoms of depression, anxiety, and PTSD. ${ }^{32-34}$ Accordingly, it remains unclear to what extend sleep changes might explain the variance in symptom improvements. Future studies should therefore assess sleep both subjectively and objectively. Fourth, no neuroendocrine assessment was performed; such assessment might have provided more insight into the underlying neuroendocrine mechanisms. ${ }^{36,37}$ Fifth, no follow-up assessment was performed; this would have allowed investigation of the longer-term impact of CBT. Last, it is possible that the present pattern of results is due to unassessed variables, which might have biased two or more variables in the same direction.

\section{Conclusion}

The present study showed that an additional and highly standardized CBT program had a favorable influence on symptoms of PTSD, depression, and anxiety, and on autobiographical memory among patients suffering from PTSD, as compared to a control group treated pharmacologically only.

\section{Acknowledgment}

We thank Nick Emler (University of Surrey, UK) for proofreading the manuscript.

\section{Disclosure}

The authors report no conflicts of interest in this work. 


\section{References}

1. American Psychiatric Association. Diagnostic and Statistical Manual of Mental Disorders. 4th ed. Washington: American Psychiatric Association; 1994.

2. Health and human rights [webpage on Internet]. Post traumatic stress disorder. 2014. Available from: http://www.hhri.org/thematic/ptsd. html.

3. Difede J, Olden M, Cukor J. Evidence-based treatment of post-traumatic stress disorder. Annu Rev Med. 2014;65:319-332.

4. van Liempt S, Vermetten E, Geuze E, Westenberg HG. Pharmacotherapy for disordered sleep in post-traumatic stress disorder: a systematic review. Int Clin Psychopharmacol. 2006;21:193-202.

5. Schoenfeld FB, Deviva JC, Manber R. Treatment of sleep disturbances in posttraumatic stress disorder: a review. J Rehabil Res Dev. 2012;49:729-752.

6. Gradus JL, Qin P, Lincoln AK, et al. Posttraumatic stress disorder and completed suicide. Am J Epidemiol. 2010;171:721-727.

7. Sjöström N, Hetta J, Waern M. Persistent nightmares are associated with repeat suicide attempt: a prospective study. Psychiatry Res. 2009;170:208-211.

8. Tanskanen A, Tuomilehto J, Viinamäki H, Vartiainen E, Lehtonen J, Puska P. Nightmares as predictors of suicide. Sleep. 2001;24:844-847.

9. Ravindran LN, Stein MB. Pharmacotherapy of post-traumatic stress disorder. Curr Top Behav Neurosci. 2010;2:505-525.

10. Polak AR, Witteveen AB, Visser RS, et al. Comparison of the effectiveness of trauma-focused cognitive behavioral therapy and paroxetine treatment in PTSD patients: design of a randomized controlled trial. BMC Psychiatry. 2012;12:166.

11. Brewin CR. Episodic memory, perceptual memory, and their interaction: foundations for a theory of posttraumatic stress disorder. Psychol Bull. 2014;140:69-97.

12. Hendriksen H, Olivier B, Oosting RS. From non-pharmacological treatments for post-traumatic stress disorder to novel therapeutic targets. Eur J Pharmacol. 2014;732:139-158.

13. Bisson JI, Ehlers A, Matthews R, Pilling S, Richards D, Turner S. Psychological treatments for chronic post-traumatic stress disorder. Systematic review and meta-analysis. Br J Psychiatry. 2007;190:97-104.

14. Ipser J, Seedat S, Stein DJ. Pharmacotherapy for post-traumatic stress disorder - a systematic review and meta-analysis. S Afr Med J. 2006;96:1088-1096.

15. Steckler T, Risbrough V.Pharmacological treatment of PTSD-established and new approaches. Neuropharmacology. 2012;62(2):617-627.

16. Mellman TA, Clark RE, Peacock WJ. Prescribing patterns for patients with posttraumatic stress disorder. Psychiatr Serv. 2003; 54(12):1618-1621.

17. Hetrick SE, Purcell R, Garner B, Parslow R. Combined pharmacotherapy and psychological therapies for post traumatic stress disorder (PTSD). Cochrane Database Syst Rev. 2010;(7):CD007316.

18. Otto MW, Hinton D, Korbly NB, et al. Treatment of pharmacotherapyrefractory posttraumatic stress disorder among Cambodian refugees: a pilot study of combination treatment with cognitive-behavior therapy vs sertraline alone. Behav Res Ther. 2003;41(11):1271-1276.

19. Schneier FR, Neria Y, Pavlicova M, et al. Combined prolonged exposure therapy and paroxetine for PTSD related to the World Trade Center attack: a randomized controlled trial. Am J Psychiatry. 2012;169(1):80-88.

20. Sutherland K, Bryant RA. Autobiographical memory and the selfmemory system in posttraumatic stress disorder. J Anxiety Disord. 2008;22(3):555-560.
21. Brown AD, Addis DR, Romano TA, et al. Episodic and semantic components of autobiographical memories and imagined future events in post-traumatic stress disorder. Memory. 2014;22(6):595-604.

22. Brown AD, Root JC, Romano TA, Chang LJ, Bryant RA, Hirst W. Overgeneralized autobiographical memory and future thinking in combat veterans with posttraumatic stress disorder. J Behav Ther Exp Psychiatry. 2013;44(1):129-134.

23. American Psychiatric Association. Diagnostic and statistical manual of mental disorders. 5th ed. Washington: American Psychiatric Association; 2013.

24. Weiss DS, Marmar CR. The Impact of Event Scale-Revised. In: Wilson JP, Keane TM, editors. Assessing psychological trauma and PTSD. 1st ed. New York City: Guilford Press; 1997. p. 399-411.

25. Beck AT, Ward CH, Mendelson M, Mock J, Erbaugh J. An inventory for measuring depression. Arch Gen Psychiatry. 1961;4:561-571.

26. Beck AT, Epstein N, Brown G, Steer RA. An inventory for measuring clinical anxiety: psychometric properties. J Consult Clin Psychol. 1988;56:893-897.

27. Kopelman MD, Wilson BA, Baddeley AD. The autobiographical memory interview: a new assessment of autobiographical and personal semantic memory in amnesic patients. J Clin Exp Neuropsychol. 1989;11(5):724-744.

28. Clark DA, Beck AT. 2011. Cognitive therapy of anxiety disorders: Science and practice. New York: Guilford Press; 2011.

29. Southwick SM, Krystal JH, Morgan CA, et al. Abnormal noradrenergic function in posttraumatic stress disorder. Arch Gen Psychiatry. 1993;50:266-274.

30. Schnurr PP, Friedman MJ, Bernardy NC. Research on posttraumatic stress disorder: epidemiology, pathophysiology, and assessment. J Clin Psychol. 2002;58:877-889.

31. Daly CM, Doyle ME, Radkind M, Raskind E, Daniels C. Clinical case series: the use of Prazosin for combat-related recurrent nightmares among Operation Iraqi Freedom combat veterans. Mil Med. 2005;170:513-515.

32. van Mill JG, Hoogendijk WJ, Vogelzangs N, van Dyck R, Penninx BW. Insomnia and sleep duration in a large cohort of patients with major depressive disorder and anxiety disorders. J Clin Psychiatry. 2010;71(3):239-246.

33. van Mill JG, Vogelzangs N, van Someren EJ, Hoogendijk WJ, Penninx BW. Sleep duration, but not insomnia, predicts the 2-year course of depressive and anxiety disorders. J Clin Psychiatry. 2014;75(2):119-126.

34. Johnson EO, Roth T, Breslau N. The association of insomnia with anxiety disorders and depression: exploration of the direction of risk. J Psychiatr Res. 2006;40(8):700-708.

35. Ahmadpanah M, Sabzeiee P, Hosseini SM, et al. Comparing the effect of prazosin and hydroxyzine on sleep quality in patients suffering from posttraumatic stress disorder. Neuropsychobiology. 2014;69(4):235-242.

36. Yehuda R, Bierer LM, Sarapas C, Makotkine I, Andrew R, Seck1 JR. Cortisol metabolic predictors of response to psychotherapy for symptoms of PTSD in survivors of the World Trade Center attacks on September 11, 2001. Psychoneuroendocrinology. 2009;34(9):1304-1313.

37. Yehuda R, Bierer LM, Pratchett LC, et al. Cortisol augmentation of a psychological treatment for warfighters with posttraumatic stress disorder: Randomized trial showing improved treatment retention and outcome. Psychoneuroendocrinology. 2015;51:589-597. 


\section{Publish your work in this journal}

Neuropsychiatric Disease and Treatment is an international, peerreviewed journal of clinical therapeutics and pharmacology focusing on concise rapid reporting of clinical or pre-clinical studies on a range of neuropsychiatric and neurological disorders. This journal is indexed on PubMed Central, the 'PsycINFO' database and CAS,

and is the official journal of The International Neuropsychiatric Association (INA). The manuscript management system is completely online and includes a very quick and fair peer-review system, which is all easy to use. Visit http://www.dovepress.com/testimonials.php to read real quotes from published authors.

Submit your manuscript here: http://www.dovepress.com/neuropsychiatric-disease-and-treatment-journal 